\title{
Atomic-Scale EELS Maps: It's Not Resolution, but Contrast
}

\author{
Huolin L. Xin, Lena Fitting Kourkoutis, ${ }^{* *}$ Julia A. Mundy, ${ }^{* *}$ Han Zheng, ${ }^{* *}$ and David A. Muller ${ }^{* *}$ \\ * Department of Physics, Cornell University, Ithaca, NY 14853 \\ ** School of Applied and Engineering Physics, Cornell University, Ithaca, NY 14853
}

The large usable current of an aberration-corrected scanning transmission electron microscope (STEM) allows for rapid acquisition of 2-D electron energy loss spectroscopic images (EEL-SI), which eliminates additional resolution degradation caused by instrumental instabilities. Enabled by this technique, multiple 2-D STEM-EELS studies have shown atomic resolution mapping using highenergy core-loss electrons [1-2] and, on the other hand, have demonstrated a significant resolution degradation or even contrast reversal when lower-energy-loss signals are used [3-5]. In this work, we, both theoretically and experimentally, demonstrate how delocalization of inelastic scattering affects the contrast of EELS maps, which allows us to determine the practical resolution limit of EELS mapping as a function of energy loss in an aberration-corrected STEM.

In 2-D EELS mapping, the signal is based upon inelastically scattered electrons and thus, in addition to the probe size, delocalization of inelastic scattering has to be taken into account when estimating the spatial resolution of this technique. To study this effect as a function of energy loss, we performed 2-D EELS mapping of Eu in $\mathrm{EuTiO}_{3}$ using a $100 \mathrm{keV}$ Nion UltraSTEM $\left(\alpha_{\max }=34 \mathrm{mrad}\right.$, $\left.\beta_{\max }=77 \mathrm{mrad}\right)$. As shown in Fig. 1, the contrast of the Eu map obtained by the Eu-M edge $(\sim 1100$ $\mathrm{eV})$ is significantly better than that obtained by the Eu-N edge $(\sim 133 \mathrm{eV})$. The degradation of contrast is qualitatively expected from the classical theory of impulse response [6]; however, to quantitatively predict it, a quantum mechanical calculation is needed. Here, we perform a full quantum mechanical simulation with the dipole approximation [7-8]. It is worth noting that, for an electron probe with a size close to the Bohr radius, the dipole approximation is not fully accurate. However, it gives an upper bound on the resolution limit as the higher orders are more localized [9]. Fig. 2a shows the simulated EELS line scan across a single atom. Because the collection angle (77 mrad) is much larger than the convergence angle (34 mrad), the full width at half maximum (FWHM) stays nearly constant throughout the whole energy range (Fig. 2b). This result at first glance seems to contradict our experimental results. However, inelastic delocalization adds a large tail to the low-energy loss EELS profile (Fig. 2a). As the energy loss approaches zero, this large tail is manifested in the divergence of the radius of the disk containing $80 \%$ of the signal $\left(r_{80 \%}\right)$ (Fig. $2 \mathrm{~b}$ ). In a real sample, where more than one atom is involved, the increase in $r_{80 \%}$ translates to a decrease in lattice contrast (or long tails at an interface), in agreement with our experimental results (Fig. 1).

In addition, the reduction in the fringe contrast can be quantified by analyzing the damping of the contrast transfer function (Fig. $3 \mathrm{a}$ and $\mathrm{b}$ ). The $2-\AA \AA \& 4-\AA$ fringe contrast falls off rapidly as the energy loss decreases (Fig. 3c). For practically microscopy, noise cannot be eliminated, which will further limit the spatial resolution. As the fringe contrast drops below the minimum detectable contrast, the lattice fringe is not visible in a resulting EELS map. Therefore, when estimating the resolution in 2D EELS mapping, one has to compare the reduced fringe contrast with the minimum detectable contrast set by the counting statistics and the signal-to-noise ratio at the energy of interest.

Moreover, in a realistic sample with a finite thickness, the inelastic contrast can be mixed with the elastic scattering, because some inelastically scattered electrons undergo multiple elastic scattering and are thus scattered outside the spectrometer. This effect could lead to a contrast reversal in the EELS mapping as shown by Wang [3]. We have experimentally observed this effect in a series EELS maps and we will show the thickness dependence of the energy at which contrast reversal happens.

\section{Reference}

[1] M. Bosman et al., Phys Rev Lett 99, 086102 (2007).

[2] D. A. Muller et al., Science 319, 1073 (2008).

[3] P. Wang et al., Physical Review Letters 101 (2008). 
[4] L. F. Kourkoutis et al., Pholosophical Magazine (Submitted).

[5] H. L. Xin, and D. A. Muller, Microscopy and Microanalysis 15 (Suppl. 2), 1198 (2009).

[6] J. D. Jackson, Classical electrodynamics 3rd edition (Wiley, New York, 1998).

[7] H. Kohl, and H. Rose, Advances in Imaging and Electron Physics 65, 173 (1985).

[8] D. A. Muller, and J. Silcox, Ultramicroscopy 59, 195 (1995).

[9] A. J. D'Alfonso et al., Ultramicroscopy 108, 677 (2008).

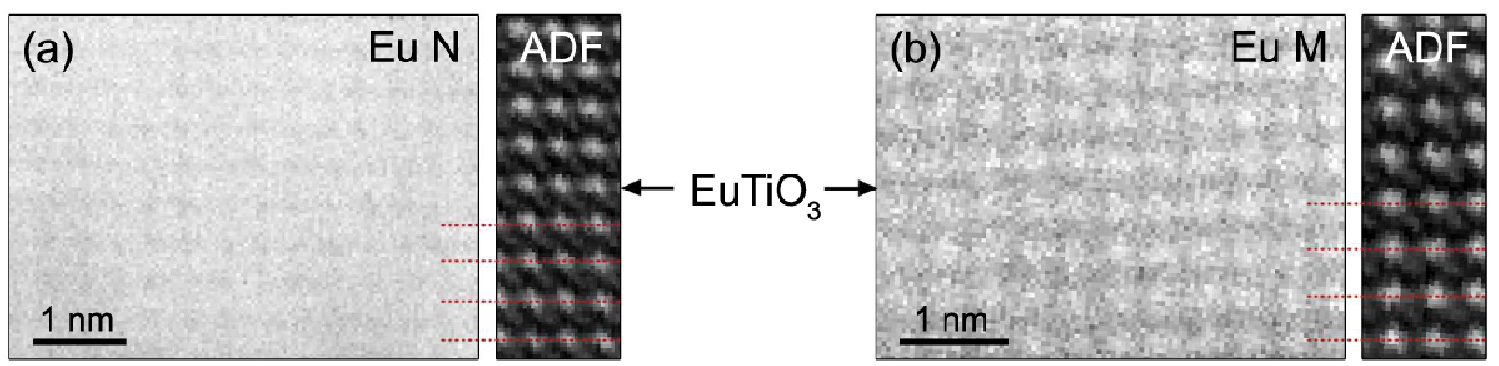

Fig. 1. Delocalization effects in atomic resolution spectroscopic imaging. (a) Eu- $\mathrm{N}_{4,5}(\sim 135 \mathrm{eV})$ and (b) Eu-M $\mathrm{M}_{4,5}$ $(\sim 1100 \mathrm{eV})$ elemental maps of $\mathrm{EuTiO}_{3}$ obtained from two individual spectroscopic images. A part of the simultaneously recorded ADF image is shown for comparison on the right side of each map.
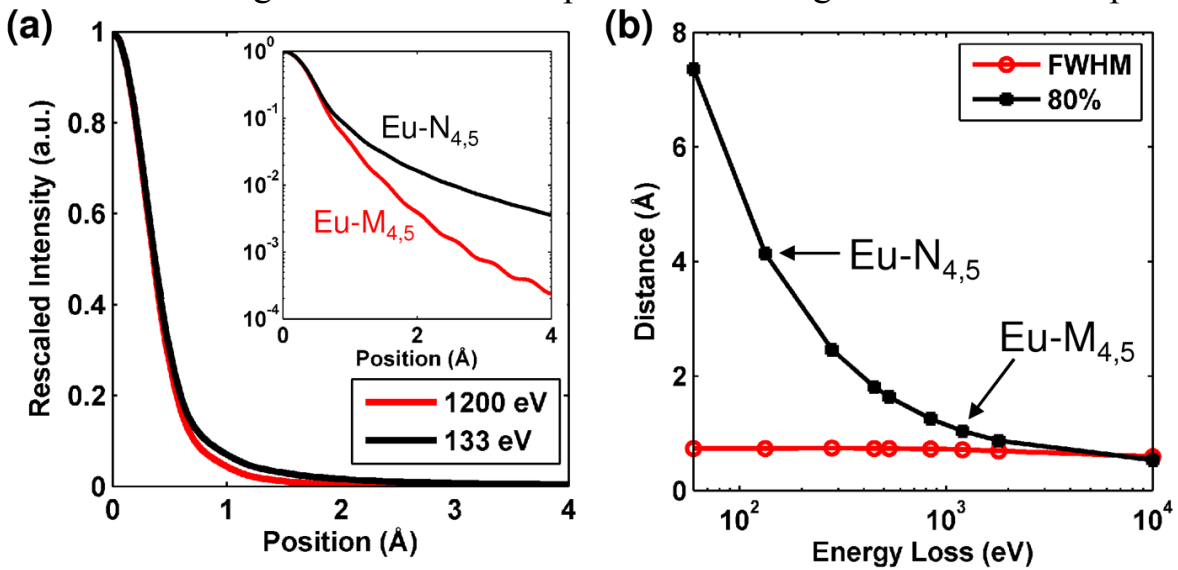

Fig. 2. Delocalization for a $100 \mathrm{kV}$ aberration-free STEM at zero defocus $\left(\alpha_{\max }=34 \mathrm{mrad}, \beta_{\max }=77 \mathrm{mrad}\right)$ calculated within the dipole approximation. (a) The simulated EELS line scans for an energy loss of $133 \mathrm{eV}$ and $1200 \mathrm{eV}$, respectively. In the inset the intensity is replotted on a log scale. (b) measures of spatial resolution as a function of energy loss: the radius of the disk containing $80 \%$ of the scattered electrons $\left(r_{80 \%}\right)$ and the full width at half maximum (FWHM).
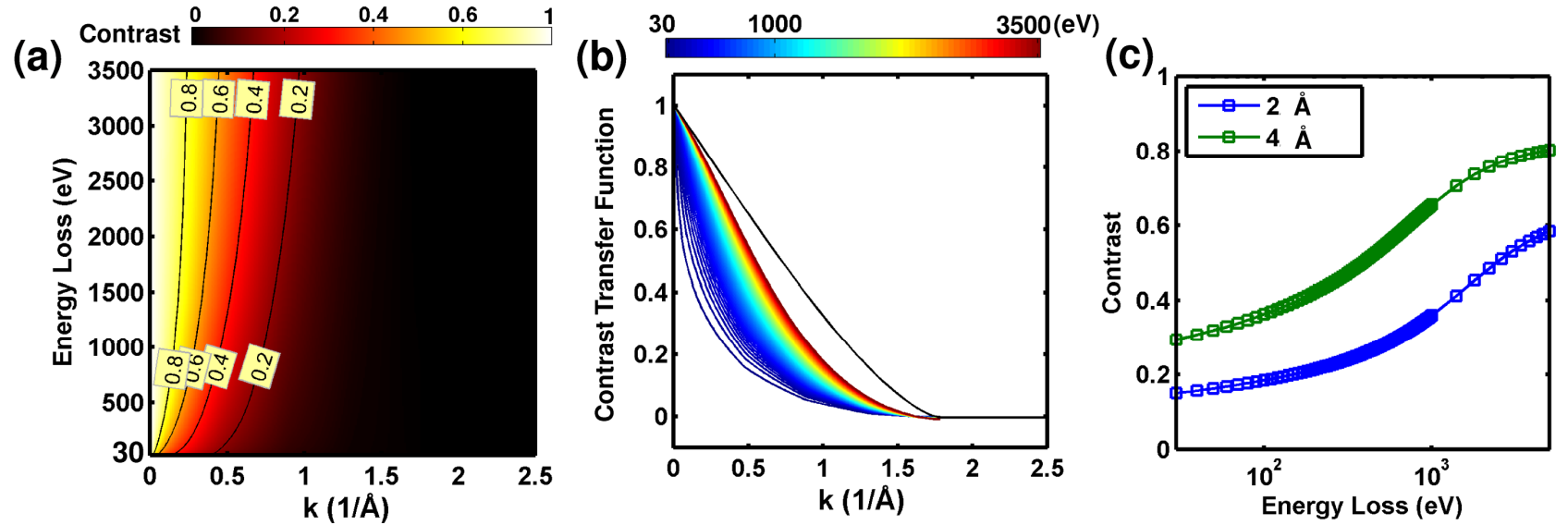

Fig. 3. Contrast of EELS mapping as a function of energy loss. (a-b) Contrast transfer function (CTF) vs. energy loss. The black line in (b) is the CTF of HAADF imaging (elastic limit). Delocalization is manifested as the damping of the CTF compared to the elastic limit. (c) The $2 \AA$ and $4 \AA$ fringes contrast in and EELS map as a function of energy loss. 